\title{
Electronic energy loss of channeled ions: The giant Barkas effect
}

\author{
L. L. Araujo, P. L. Grande, M. Behar, and J. F. Dias \\ Instituto de Física, Universidade Federal do Rio Grande do Sul, Caixa Postal 15051, CEP 91501-970, Porto Alegre (RS), Brazil \\ A. F. Lifschitz and N. R. Arista \\ División Colisiones Atómicas, Centro Atómico Bariloche, Instituto Balseiro, RA-8400, San Carlos de Bariloche, Rio Negro, Argentina \\ G. Schiwietz \\ Hahn-Meitner-Institut, Bereich Festkörperphysick, Glienicker Strasse 100, 14109 Berlin, Germany
}

(Received 20 April 2004; published 22 September 2004)

\begin{abstract}
In this work we have measured the electronic energy loss of ${ }^{9} \mathrm{Be}$ and ${ }^{11} \mathrm{~B}$ ions for the $\langle 100\rangle$ and $\langle 110\rangle$ directions in $\mathrm{Si}$ as a function of the incident ion energy. The channeling measurements cover a wide energy range between $100 \mathrm{keV} / \mathrm{amu}$ and $1 \mathrm{MeV} / \mathrm{amu}$. The Rutherford backscattering technique has been employed in the present experiments. An overall compilation of channeling energy loss values for several ions, including those for $\mathrm{He}, \mathrm{Li}$, and $\mathrm{O}$ measured previously, provides a clear picture of the Barkas contribution to the stopping power due to valence electrons in the present energy regime. A maximum of the relative contribution occurs for Be ions around $250 \mathrm{keV} / \mathrm{amu}$ while a saturation effect is observed for heavier ions. These results are interpreted in terms of a self-consistent nonlinear calculation based on the transport cross-section approach together with the unitary convolution approximation model, which describe the present data reasonably well at high projectile energies.
\end{abstract}

DOI: 10.1103/PhysRevA.70.032903

PACS number(s): 34.50.Bw, 61.85.+p, 34.50.Fa, 79.20.Rf

\section{INTRODUCTION}

The measurement of the energy loss of light projectiles under axial channeling conditions is of primary interest in order to improve the understanding of fundamental ion-solid interactions. Channeled ions attain a very particular distribution along the channel, being pushed towards its center, giving rise to what is known as flux peaking [1]. As a consequence, the incoming ion interacts mostly with valence electrons of the crystal under study, providing a relatively simple scenario where, for instance, an electron gas model is realized and auxiliary effects such as shell corrections and electron capture play a minor role in the interaction of the ions with the crystal. Therefore, the study of polarization effects in ionizing collisions becomes attractive, since the theoretical approach is simplified in this case.

The Barkas effect, in particular, accounts for the difference in the stopping powers of particles and their respective antiparticles and was first observed by Barkas when studying hyperon masses [2]. Barkas himself suggested that this difference might stem from higher-order terms relative to the Bethe theory [3] (namely, from the interference between the first- and second-order terms). At lower energies and for projectiles carrying bound electrons, the definition of the Barkas effect is somewhat controversial [4] due to the interplay of other effects. In this work, it is assumed that the Barkas effect stands for the different way the electron cloud is polarized when identical ions (with the same internal structure) but with opposite charges pass by. Other polarization effects also appear in multiple ionizing collisions due to highly charged projectiles and have been recently investigated for gas targets $[5,6]$.

Initially, the observation of the Barkas effect relied upon indirect measurements only, where the ranges of positively charged particles in emulsions were observed to be smaller than those of negatively charged ones [2]. Later on, with the advent of antiproton beams, a direct measurement of the Barkas effect was possible using both proton and antiprotons beams $[7,8]$. Although some attempts were made for direct measurements using ion beams, it was found that the Barkas contribution to the stopping power in such cases was extremely small and difficult to quantify [9].

Recently, after some attempts [10,11], the channeling technique was proven to be a powerful tool to extract the Barkas contribution to the valence stopping power from experimental data. Indeed, it has been shown [12] that for $\mathrm{He}$ and $\mathrm{Li}$ ions traveling in axial channels in $\mathrm{Si}$, the Barkas effect is responsible for a sizable fraction of the total stopping power. For $\mathrm{Li}$ ions in $\mathrm{Si}$ along the $\langle 110\rangle$ direction, this contribution amounts to $50 \%$, characterizing what has been termed as the giant Barkas effect [12]. Later a similar study was carried out for $\mathrm{O}$ ions channeling in the $\mathrm{Si}\langle 100\rangle$ direction [13]. In this case, the relative contribution of the Barkas effect to the stopping power was found to be smaller than those observed for $\mathrm{He}$ and $\mathrm{Li}$ ions in the same channel in $\mathrm{Si}$ at low velocities.

At this point, it became clear that the gap between $\mathrm{Li}$ and $\mathrm{O}$ projectile should be filled, in order to provide a clear picture of the behavior of the absolute and relative contributions of the Barkas effect to the stopping power. In particular, the question whether there is a smooth behavior of these contributions should be clarified by new measurements. Therefore, we have embarked on measurements of the channeling stopping power of ${ }^{11} \mathrm{~B}$ and ${ }^{9} \mathrm{Be}$ in $\mathrm{Si}\langle 100\rangle$ and $\langle 110\rangle$ directions as a function of the ion energy. While ${ }^{11} \mathrm{~B}$ has been measured along the $\mathrm{Si}\langle 100\rangle$ previously $[14,15]$, this is the first measurement of ${ }^{9} \mathrm{Be}$ under channeling conditions in $\mathrm{Si}$. The the- 


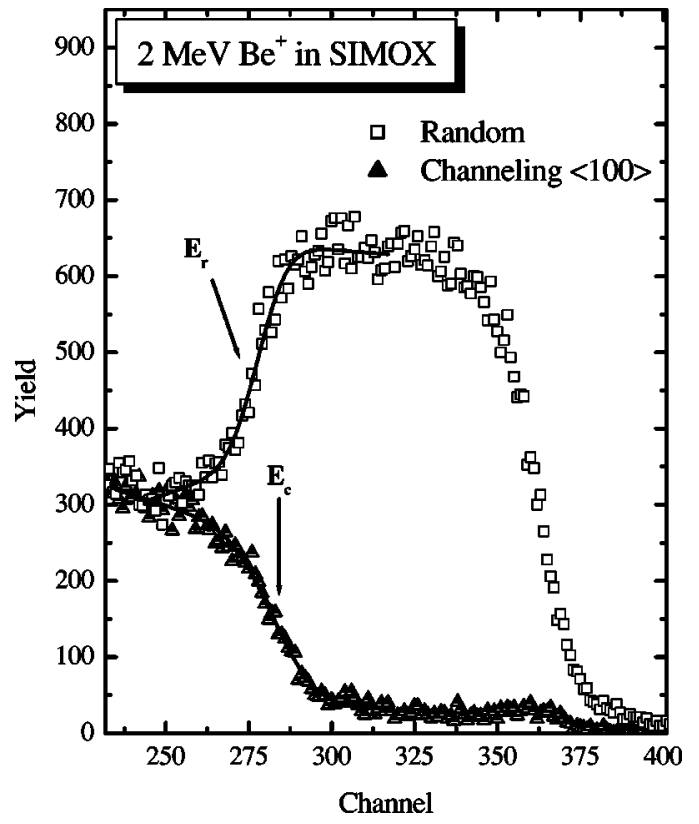

FIG. 1. RBS spectra for $2-\mathrm{MeV}^{9} \mathrm{Be}^{+}$ions in a nonaligned geometry and channeled in the $\langle 100\rangle$ axial direction in SIMOX. $E_{c}$ and $E_{r}$ stand for the detected energies of the particles backscattered at the $\mathrm{Si} / \mathrm{SiO}_{2}$ interface for channeling and random (nonaligned) conditions, respectively. Lines represent the results of the fitting procedure [19] used to determine the corresponding energy loss.

oretical background used to interpret the data relies on a binary collision approximation combining self-consistent nonlinear calculations based on the transport cross-section approach [16] with the unitary convolution approximation to describe the impact-parameter dependence of the energy loss [17]. Atomic units (a.u.) will be used throughout the text unless otherwise stated.

\section{EXPERIMENTAL AND ANALYSIS PROCEDURES}

For the channeling experiments we have used SIMOXtype samples consisting of $2000-\AA \mathrm{Si}\langle 100\rangle$ crystal layers on top of $4000-\AA \mathrm{SiO}_{2}$ buried layers, produced in $\langle 100\rangle \mathrm{Si}$ wafers. Given the quality of the $\mathrm{Si} / \mathrm{SiO}$ interface, it was possible to develop a simple method to extract the channeling stopping power of ions in Si from their respective Rutherford backscattering (RBS) measurements [19]. The backscattered particles were detected by $\mathrm{Si}$ surface-barrier detectors located at $170^{\circ}$ with respect to the incident beam. The overall resolution of the detection system was about 20 and $23 \mathrm{keV}$ for beryllium and boron, respectively. Typical RBS spectra are shown in Fig. 1 for Be ions with primary energy $E_{0}$ impinging on $\mathrm{Si}\langle 100\rangle$ and for random directions. In this case, the random direction is defined according to the procedure developed by Dygo et al. [20], which takes as references the $\langle 100\rangle$ axis $(\Psi=0)$ and the $\{100\}$ plane $(\phi=0)$, leading to a random direction defined by $\Psi=6^{\circ}$ and $\phi=15^{\circ}$. In Fig. 1, $E_{c}$ and $E_{r}$ stand for the detected mean energies of the backscattered particles at the $\mathrm{Si} / \mathrm{SiO}_{2}$ interface for the channeled and random incidence, respectively. These energies were determined by fitting the channeling and random spectra with an algorithm [19] which includes, in addition to the error function (accounting for particle straggling and detector resolution), the Rutherford cross-section dependence on the energy. Finally, in the framework of the mean energy approximation, it can be shown [19] that the specific channeling energy loss along the incoming projectile path (before the backscattering) is a function of the energies $E_{0}, E_{c}$, and $E_{r}$ of the specific energy loss in the random direction and of geometrical factors. For this particular purpose, the specific energy loss of beryllium and boron in random directions has been already measured and presented elsewhere [21,22].

At this point, it is important to stress that the channeling stopping power depends indeed on the target thickness because of the variation of the ion-flux distribution and projectile charge state. For very thin crystals, the ion-flux distribution is nearly uniform and the projectile charge state is equal to the incident one. After less than a few hundred nanometers, equilibrated ion-flux distributions and projectile charge states are achieved. Then, for a thin film, assuming that the effect of the preequilibrium projectile charge state is of minor importance (because an incident charge state close to the equilibrium value can be chosen), the channeling stopping power will be nearly identical to the random one. On the other hand, if the crystal is too thick, the channeling stopping power also may approach the random one due to dechanneling at crystal defects, thermal vibrations, and electronic multiple scattering. For the present experimental conditions (light ions at kinetic energies of a few hundred $\mathrm{keV}$ per nucleon), SIMOX targets of $2000 \AA$ can be considered thick enough to prevent preequilibrium ion-flux distributions as well as thin enough to avoid enhanced dechanneling effects.

\section{THEORETICAL FORMULATION}

Under channeling conditions, the energy loss due to the $\mathrm{Si}$ inner-shell electrons is strongly suppressed, since the ionflux distribution along a Si major axial direction has a peak in the middle of the channel (flux peaking). With the exception of the widest channel of Si (the $\mathrm{Si}\langle 110\rangle$ channel), there is nearly no influence of the flux distribution on the valenceelectron energy loss, since the distribution of the valence electrons across the channel varies only by a few percent [23]. Therefore, measurements of the electronic energy loss along the $\mathrm{Si}\langle 100\rangle$ axial direction are representative of an electron-gas system (featured by an electron radius $r_{s}=2$ ).

Here we will use a combination of two theoretical approaches to compare to the experimental $\langle 100\rangle$ channeling data-namely, the HISTOP [16] self-consistent nonlinear calculations based on the transport cross-section approach and the unitary convolution approximation (UCA) $[17,18]$. The first approach is one of the few models that provides the Barkas effect in a nonperturbative way (another example of a nonperturbative approach is the binary model described in Ref. [24]) but relies on the homogeneous electron-gas approximation. The second model has been used [12] to extract the Barkas effect from the experimental data, since it accounts for other higher-order terms and for the crystal structure very accurately $[17,25]$. We have also used the coupled- 
channel method $[26,27]$ to calculate the electronic energy loss for the $\mathrm{Si}$ inner-shell electrons. This time-consuming approach takes into account fully the Barkas effect as well as all other higher-order effects for inner-shell electrons.

\section{A. Nonlinear stopping approach}

The self consistent nonlinear model to represent the energy loss of nonrelativistic ions was derived in previous papers, both for light and heavy ions [28,29]. The target is considered as a free-electron-like medium, and the energy loss process is described on the basis of the following assumptions: (a) the extended Friedel sum rule (EFSR), used as a procedure to optimize the screening potential in a selfconsistent way for each ion velocity, and (b) the transport cross-section method (TCS) to calculate the energy transfer from the moving ion to the valence electrons of the target. The scattering calculations are made using the phase-shift method, by numerically solving the radial Schrödinger equation for the electron-ion scattering process. These procedures have been condensed in a recently developed program that incorporates a practical software to evaluate the HISTOP [16] due to their interaction with the valence electrons in solids.

The definition of the Barkas effect for bare ions at high projectile energies is pretty clear by considering either the difference between the stopping of particles and antiparticles or the difference between the particle stopping and the Bloch formula. Both methods result in an identical leading term proportional to $Z^{3}$, purely due to polarization effects (here when referring to polarization we include, in a general context, the nonlinear effects arising from close collisions, as well as the long-range target polarization).

However, at intermediate and lower velocities the ions are no longer bare, and therefore the first procedure is no longer appropriate as a mean to obtain the polarization contribution to the stopping (what we denote by Barkas polarization effect). The corresponding difference between the stopping cross sections for a particle and its antiparticle at charge-state equilibrium is not adequate for this purpose, because it mixes two effects: screening by bound electrons and polarization.

Therefore, we calculate the Barkas polarization stopping power $\left[(d E / d x)_{\text {Barkas }}\right]$ by reversing the sign of the interacting potential $V$ (even for screened ions), according to

$$
\left(\frac{d E}{d x}\right)_{\text {Barkas }}=0.5\left(\frac{d E}{d x}(V)-\frac{d E}{d x}(-V)\right) .
$$

In this way, all even higher-order terms in a $Z$ expansion are removed at high energies and only the odd (polarization) terms are kept for all ion energies. Although measurements involving an ion with reversed interacting potential or an "antiparticle carrying bound positrons" are not feasible, the use of Eq. (1) avoids the mixture of effects and leads to a clear theoretical determination of the polarization effect at any energy. Moreover, in order to avoid any further semantic misunderstandings, in what follows we will use the term Barkas-polarization effect instead of Barkas effect.

\section{B. Unitary convolution approximation}

A detailed description of the UCA model for bare and screened ions may be found elsewhere $[17,18]$ and imple- mented in Ref. [30]. This model provides a simple formula for the impact-parameter-dependent energy loss realizing the Bloch formula [31] for bare ions at high velocities. The formula for the energy transfer $Q$ at a given impact parameter $b$ reads

$$
Q(b)=\int d^{2} r_{T} \mathcal{K}\left(\vec{b}-\vec{r}_{T}\right) \int d z \rho\left(\vec{r}_{T}, z\right),
$$

which is a convolution of the electronic density $\rho$ integrated along the ion path (along the $z$ direction perpendicular to $\vec{r}_{T}$ ) with a given kernel $\mathcal{K}$ [17] that interpolates distant and close collisions smoothly and depends on the projectile screening and target oscillator-strengths [17]. Recently, this model has been extended to include shell corrections [32]. Thus, the major limitations of this model are the absence of electron capture and polarization effects. Nevertheless, accurate coupled-channel benchmark calculations have shown [17] that other higher-order effects are very well described in this simple approach.

The mean energy lost by the projectile after passing a certain thickness $t$ is given by

$$
\Delta E=\frac{\int_{\mathcal{A}} d^{2} r_{c} \int_{0}^{t} d z \frac{d E\left(\vec{r}_{c}\right)}{d z} \Phi\left(\vec{r}_{c}, z\right)}{\int_{\mathcal{A}} d^{2} r_{c} \int_{0}^{t} d z \Phi\left(\vec{r}_{c}, z\right)},
$$

where $\mathcal{A}$ is the transversal area of the $\mathrm{Si}$ axial channel, $\vec{r}_{c}$ is the position relative to the center of the channel, and $\Phi\left(\vec{r}_{c}, z\right)$ is the ion flux distribution at the distance $\vec{r}_{c}$ and channeling direction $z$. The energy loss per traversed distance $(d E / d z)\left(\vec{r}_{c}\right)$ may be divided into two contributions:

$$
\frac{d E}{d z}\left(\vec{r}_{c}\right)=\left(\frac{d E}{d z}\left(\vec{r}_{c}\right)\right)_{\text {direct }}+\left(\frac{d E}{d z}\left(\vec{r}_{c}\right)\right)_{\text {e loss }} .
$$

The first contribution corresponds to the energy loss involving the Si electrons, while the second one is due to energy loss from electrons originally bound to the projectile (projectile ionization and excitation). Both contributions are obtained from Eq. (2) for a projectile with charge state $q$ and a neutral $\mathrm{Si}$ atom. Finally, an average over the projectile charge states $q$ has to be performed. The B charge-state distributions were taken from experiments performed under channeling conditions [15] and extrapolated down to $100 \mathrm{keV} /$ nucleon. For Be ions, an interpolation procedure has been carried out using the experimental values of charge fractions for elements close to $\mathrm{Be}$ [15]. We have also used semiempirical charge-state fractions obtained from the CASP program [30].

In order to obtain the ion flux distribution, we have used standard Monte Carlo calculations [33] with thermal vibrations given by $\sigma=0.083 \AA$ (root mean square of thermal displacements) according to the Debye temperature $T=490 \mathrm{~K}$ extracted from the work of Dygo and collaborators [20].

\section{Coupled-channel method}

Coupled-channel (CC) calculations are the best tool to describe inner-shell ionization and excitation of atoms 


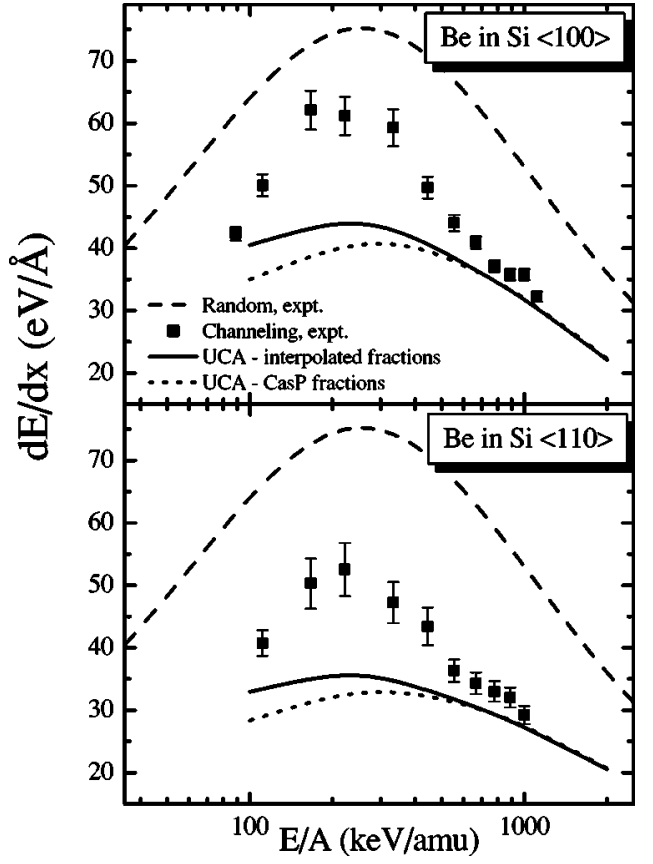

FIG. 2. Electronic stopping power of ${ }^{9} \mathrm{Be}$ ions as a function of energy for the $\langle 100\rangle$ and $\langle 110\rangle$ directions in Si. Solid squares: this work. Dashed line: measured random stopping [21]. Solid line: UCA model with the interpolation approach for the charge-state fractions. Dotted line: UCA model with charge-state fractions from the CASP program [30]. The Barkas-polarization enhancement is given by the difference in the $d E / d x$ value between the squares and solid line.

$[26,27]$ as a function of the impact parameter. These timeconsuming calculations are based on the semiclassical method [34]. The projectile following a classical trajectory provides a time-dependent electrostatic perturbation on the target electrons. Hence, the time-dependent Schrödinger equation is solved by expanding the electronic wave function in a truncated basis of states-namely, atomic orbitals. A set of first-order ordinary coupled differential equations for the coefficients originating from this expansion, the so-called coupled-channel equations, is integrated numerically along the classical trajectory of the projectile for a given impact parameter $b$. For inner-shell electrons the projectile screening (due to bound electrons) plays a minor role and thus the active-electron interaction is just the Coulomb potential. Details of the coupled-channel calculations for the Si subshells may be found elsewhere [35].

\section{RESULTS AND DISCUSSIONS}

According to the procedure outlined in Sec. II, we have determined the energy loss of ${ }^{9} \mathrm{Be}$ and ${ }^{11} \mathrm{~B}$ ions along the $\mathrm{Si}\langle 100\rangle$ and $\langle 110\rangle$ directions. These results are presented in Figs. 2 and 3. In the case of B and the $\langle 100\rangle$ direction, other channeling data measured by Jiang et al. [15] and dos Santos et al. [14] are displayed as well. The quoted errors have been estimated from several measurements. The stopping powers at random directions [21,22] (which have been used as input to the data analysis) are also shown in Figs. 2 and 3. An

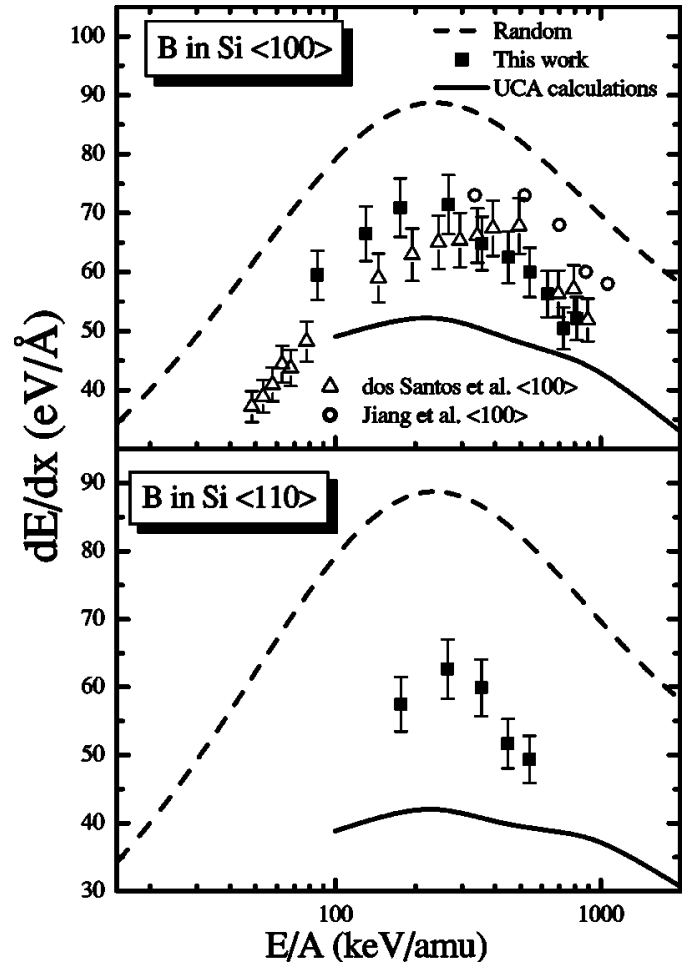

FIG. 3. Electronic stopping power of ${ }^{11} \mathrm{~B}$ ions as a function of energy for the $\langle 100\rangle$ and $\langle 110\rangle$ directions in Si. Solid squares: this work. Dashed line: measured random stopping [22]. Solid line: UCA model. Open circles: channeling data by Jiang et al. [15]. Open triangles: channeling data by dos Santos et al. [14]. The Barkas-polarization enhancement is given by the difference in the $d E / d x$ value between the squares and solid line.

inspection of these figures indicates that the channeling stopping powers are about $20 \%$ (for the $\langle 100\rangle$ direction) and $30 \%$ (for the $\langle 110\rangle$ direction) lower than the random results for both ions around the stopping maximum. Moreover, the relative difference increases with increasing ion energy. This behavior reflects the role of the valence and inner-shell electrons. Indeed, for increasing projectile energies, the contribution of the inner-shell electrons to the random stopping power increases (influence of the kinematical ionization threshold), whereas the contribution from these electrons is strongly suppressed under axial channeling conditions. The variation of the ion-flux distribution has a counteracting but very small influence on the total channeling energy loss.

A comparison of our results for B with previous experimental channeling data (open symbols) [15], obtained using the transmission technique, is also depicted in Fig. 3. As can be observed, the channeling experimental data by Jiang et al. [15] are systematically larger (by 10\%-20\%) than the present experimental results. This fact probably comes from the fact that transmission-channeling data by Jiang et al. have been measured employing thick targets (about $10000 \AA$ ), which may introduce an overestimation of the channeling energy loss due to dechanneling at crystal defects and electronic multiple scattering.

The results of the UCA model are also presented in Figs. 2 and 3. In the case of Be ions we show the UCA results for two charge-state distributions (solid and dotted lines) accord- 


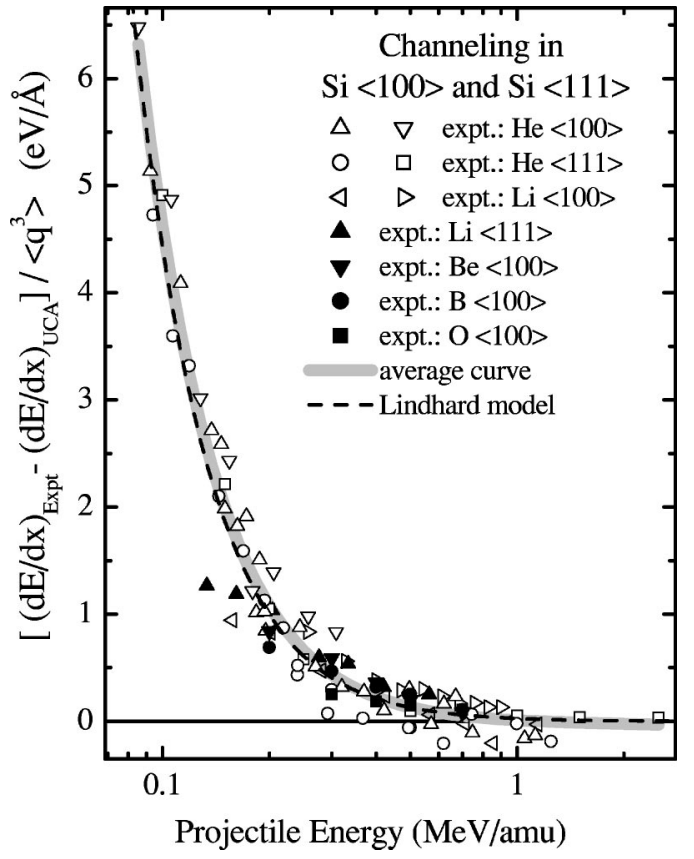

FIG. 4. Compilation of experimental channeling data $(\mathrm{He}, \mathrm{Li}$, $\mathrm{Be}, \mathrm{B}$, and $\mathrm{O}$ ) for the $\langle 100\rangle$ and $\langle 111\rangle$ directions in Si after subtraction of the UCA results and division by the mean third power of the projectile charge state as a function of the projectile energy. Data taken from Azevedo et al. [25] (open up triangles, open circles, open left triangles, solid up triangles), Lulli et al. [37] (open down triangles), Eisen et al. [36] (open squares), Jiang et al. [15] (open right triangles), Araujo et al. [13] (solid squares), and this work (solid down triangles, filled circles).

ing to Sec. III B. The uncertainties of the charge-state fractions on the UCA calculations amount to $10 \%$ at lower energies. As shown in these figures, the UCA calculations are in better agreement with the data at higher energies. Conversely, for energies around 200-500 $\mathrm{keV} / \mathrm{amu}$, the UCA calculations strongly underestimate the measured stoppingpower values. It has been shown [12] that this difference, amounting to about $40 \%$ for Be and $30 \%$ for B, comes from polarization effects which are not included in the calculations. These differences are similar to those observed for $\mathrm{Li}$ [12] and show that the giant Barkas-polarization enhancement is extended to those ions with atomic number close to $\mathrm{Li}$. In what follows we will discuss only the $\mathrm{Si}\langle 100\rangle$ direction data because the nonlinear calculations (HISTOP) are only strictly valid when a homogeneous electron gas is realized.

Here, the determination of the Barkas-polarization enhancement relies, as performed previously $[12,13]$, on the difference of the experimental channeling stopping data and the corresponding UCA calculations. Figure 4 legitimizes this approach, by showing Be and B data measured in this work together with other experimental channeling data $(\mathrm{He}$, $\mathrm{Li}$, and $\mathrm{O}$ ) for $\mathrm{Si}\langle 100\rangle$ and $\langle 111\rangle$ directions measured previously $[13,15,25,36,37]$ (see the figure caption for details). The UCA calculations have been redone here to include shell corrections and to improve the projectile screening of $\mathrm{O}$ ions with DHFS screening functions [18]. In the figure, the UCA

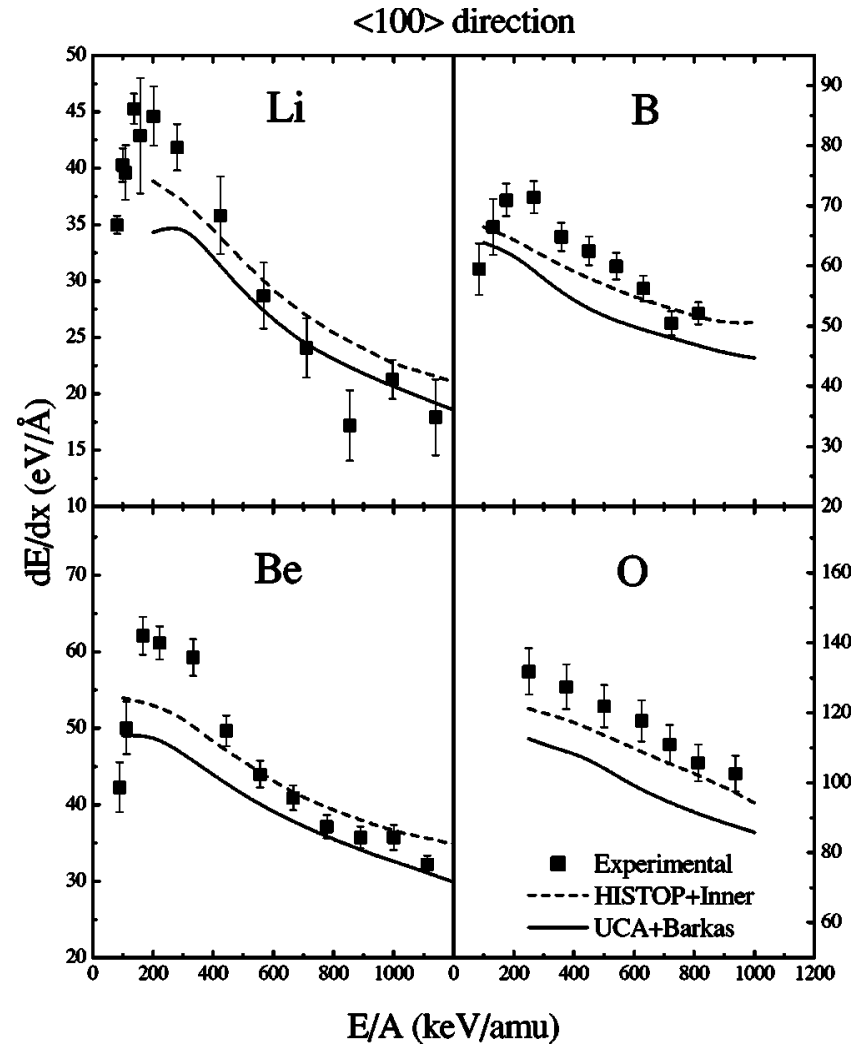

FIG. 5. Experimental channeling data for $d E / d x$ as a function of projectile energy per nucleon compared to the theoretical models. Symbols: experimental data. Solid lines: UCA calculations for the all electrons added with the Barkas valence contributions obtained from HISTOP. Dashed lines: full HISTOP calculations for the valence band added with inner-shell and electron loss contributions from UCA. See text for further details.

values were subtracted from the data and the results were divided by the mean third power of the projectile charge state. This scaled Barkas-polarization term is plotted as a function of the projectile velocity. An inspection of the figure shows that most data collapse into a narrow band (determined by a best fit) independent of the projectile species. The average curve is close to the prediction of the Lindhard model [38] (dashed curve) and Pitarke calculations [39]. It must be pointed out that we also could have divided the data in Fig. 4 by the projectile nuclear charge state to the third power $\left(Z^{3}\right)$. Even in this case, we obtain the data well grouped around an average curve. However, the scaling factor $\left\langle q^{3}\right\rangle$ is more meaningful since it takes into account different projectile charges.

As can be observed from Fig. 4 there are some remaining deviations at energies around $0.15-0.3 \mathrm{MeV} / \mathrm{amu}$. For each projectile there is a low-energy cutoff beyond which the data fall below the average curve. The cutoff energy increases strongly with the projectile nuclear charge. This clearly indicates a breakdown of the $q^{3}$ scaling and the influence of other higher-order terms in the polarization.

Figure 5 shows the experimental channeling data as function of the projectile energy for $\mathrm{Li}, \mathrm{Be}, \mathrm{B}$, and $\mathrm{O}$ ions in comparison with theoretical results discussed in Sec. III. The data for $\mathrm{Li}$ and $\mathrm{O}$ were published previously $[13,25]$ and are 


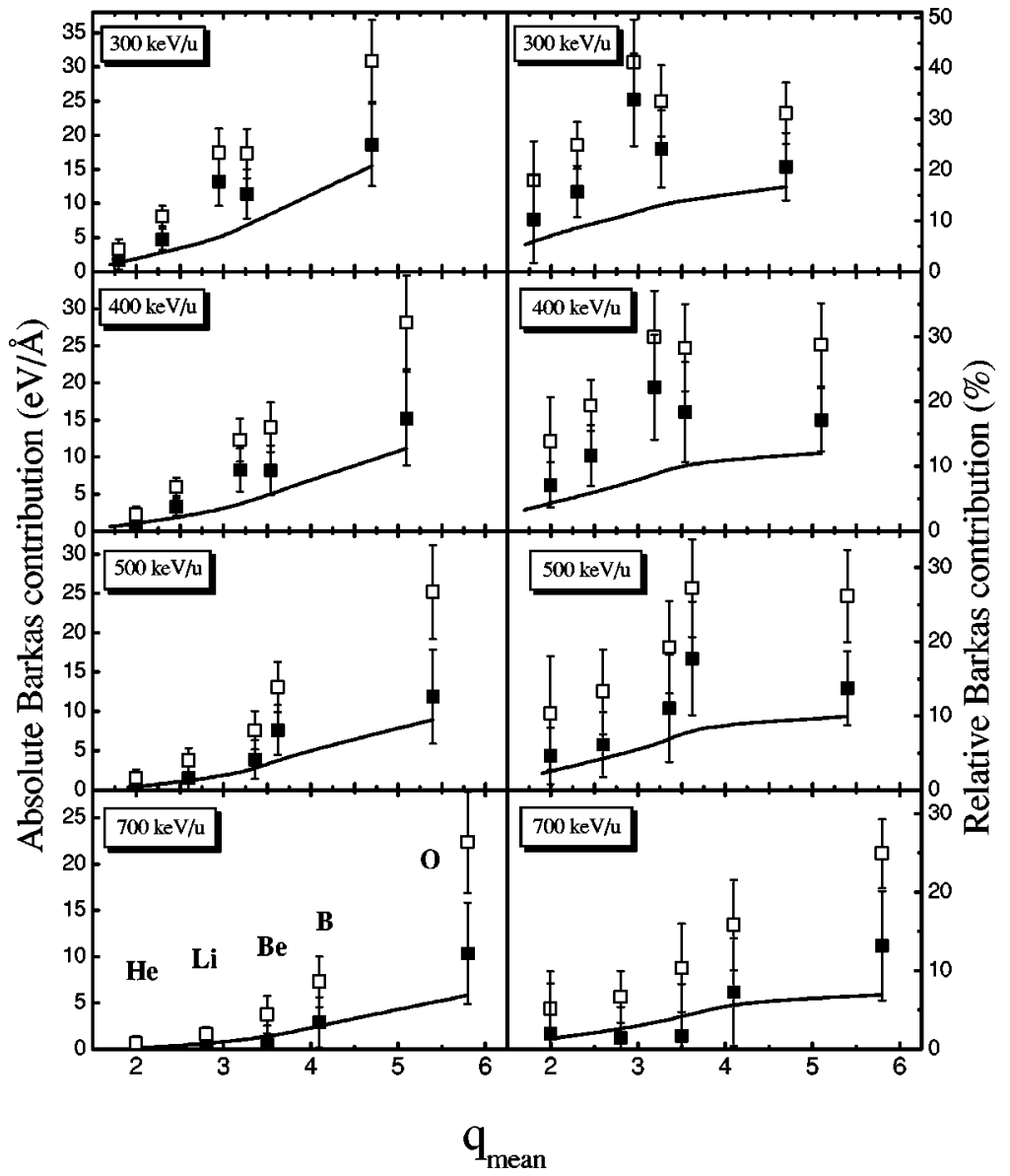

FIG. 6. Absolute (on the left panel) and relative (on the right panel) values of the Barkaspolarization contribution to the stopping power as a function of the mean charge state at four different energies per nucleon. Open squares: experimental minus full UCA results. Solid squares: experimental data minus UCA-CC calculations. Lines: Barkas calculations from HISTOP. included here only for the sake of completeness. The solid curves correspond to the UCA results plus the Barkas contribution obtained by the nonlinear calculations HISTOP through the method of charge reversal. The agreement with the experimental data is quite good at high $\mathrm{Li}$ and $\mathrm{Be}$ energies but deviations are observed for $\mathrm{B}$ and $\mathrm{O}$ ions. The experimental results can also be compared (see dashed lines) to results of the nonlinear calculations HISTOP by adding the contribution of the inner shells and electron loss from the UCA calculations. This method yields a much better agreement, in particular for $\mathrm{O}$ ions, but seems to overestimate the data at higher projectile energies for $\mathrm{Li}$ and $\mathrm{Be}$. The difference between these two theoretical approaches comes from the treatment of the valence electrons. The first model (solid line) uses a much better description of the spatial dependence of the valence-electron energy loss (through the impact parameter dependence and ion-flux distribution), while the second avoids the use of the method of charge reversal to calculate the Barkas effect (the polarization effect is inherent to the model) but relies on the homogeneous electron-gas picture.

The Barkas-polarization effect obtained from $\mathrm{He}, \mathrm{Li}, \mathrm{Be}$, $\mathrm{B}$, and $\mathrm{O}$ experimental channeling data are shown in Fig. 6 for four different velocities. Since in this energy range all these ions have many non-negligible charge states, we have plotted all curves as a function of the mean charge state $q_{\text {mean }}$. The left panel shows the absolute Barkas enhancement while the right one presents the relative effect. The open squares correspond to the experimental data after subtraction of the UCA results and represent the Barkas-polarization effect due to the valence as well as inner-shell electrons. The quoted error bars account for the experimental and theoretical (effect of different charge-state distributions in the case of $\mathrm{Be}$ ) uncertainties.

The Barkas-polarization contribution due to the $\mathrm{Si}$ inner shells is quite large (because of its large binding energy) but it is only important for close collisions and plays a minor role for ions under channeling conditions. For the present case the $\mathrm{Si}$ inner shells contribute less than $10 \%(8 \%$ for $400 \mathrm{keV} / \mathrm{u} \mathrm{B}$ ions). Nevertheless, in order to keep the focus on the valence electrons only, we have performed full $\mathrm{CC}$ calculations (according to Sec. III C) of the mean energy loss $Q(b)$ for each inner $L$ subshell of $\mathrm{Si}$. The corresponding $d E / d x$ [through Eq. (3)] has been added to the UCA results for the valence electrons and electron loss. This procedure, named UCA-CC, was used to provide theoretical stoppingpower values which have been removed from the experimental data. Then, the present experimental data minus the UCA-CC results (the solid squares in Fig. 6) should deliver the Barkas-polarization effect due to the valence electrons only. It should be stressed that the energy loss results from the coupled-channel calculations for the Si inner-shell electrons are much larger than the ones from the UCA calculations not only because of polarization effects but also due to the capture of $L$-shell target electrons into projectile bound states.

As discussed above, the solid squares as well as the solid lines, which correspond to the nonlinear Barkas calculations 
from Sec. III A, in Fig. 6 concern the valence electrons only. As can be observed, the experimental Barkas data (on the left panel) seems to increase with the mean charge state and the solid lines reproduce the experimental points quite well at larger energies $(E \geqslant 500 \mathrm{keV} /$ nucleon) but underestimate the $\mathrm{Li}, \mathrm{Be}$, and $\mathrm{B}$ data at lower energies.

The relative Barkas-polarization contribution (right panel in Fig. 6) at high energies increases with ion charge since the perturbative channeling stopping power and the lowest-order Barkas contribution increase with different power laws $q^{2}$ and $q^{3}$, respectively.

Nevertheless, at the nonperturbative regime they increase with the mean charge state at different rates. The relative Barkas-polarization effect tends to saturate within the framework of the nonlinear calculations. The experimental points follow the same trend but a small maximum can be observed at $300 \mathrm{keV} /$ nucleon for Be. The corresponding value of the Barkas contribution is about $40 \%$ at $300 \mathrm{keV} /$ nucleon and thus even larger than the one for Li reported recently in Ref. [12].

\section{CONCLUSIONS}

In the present work, we have measured the channeling stopping power of ${ }^{9} \mathrm{Be}$ and ${ }^{11} \mathrm{~B}$ along the $\mathrm{Si}\langle 100\rangle$ and $\langle 110\rangle$ directions. For the channeling measurements we have used the RBS technique associated with a SIMOX target. The main point ofthis work is the determination of the Barkaspolarization effect for the $\mathrm{Si}$ valence electrons with channeled light and heavy projectiles. In the analysis, we have used the combination of the channeling RBS method and the recent theoretical realization of the impact-parameter dependence of the Bethe-Bloch contribution (the UCA calculations). We have also used the recent development of selfconsistent nonlinear calculations for valence-band electrons. These energy losses for screened ions and their respective image (inverting interaction sign) yield the Barkaspolarization effect in the regime where the projectiles are screened by bound electrons.

At high energies the Barkas-polarization enhancement for the valence electrons is well described by the nonlinear HISTOP calculations. However, the maximum polarization enhancement (mainly for Be ions) could not be reproduced by the HISTOP calculations. This may indicate a limitation of the present Barkas valence-electron calculations which are based on two-body scattering under central forces.

\section{ACKNOWLEDGMENTS}

This work was partially supported by the Brazilian agencies CNPq and CAPES and by the cooperation programs PROBRAL 166/04 and SETCIP/CAPES 33/01.
[1] D. V. Morgan, Channeling: Theory, Observation and Applications (Wiley, New York, 1973); D. S. Gemmel, Rev. Mod. Phys. 46, 129 (1974).

[2] W. H. Barkas, N. J. Dyer, and H. H. Heckmann, Phys. Rev. Lett. 11, 26 (1963).

[3] H. Bethe, Ann. Phys. (Leipzig) 5, 324 (1930).

[4] P. Sigmund and A. Schinner, Nucl. Instrum. Methods Phys. Res. B 212, 110 (2003).

[5] M. Schulz, R. Moshammer, W. Schmitt, H. Kollmus, R. Mann, S. Hagmann, R. E. Olson, and J. Ullrich, J. Phys. B 32, L557 (1999).

[6] H. Schoene, R. Schuch, S. Datz, M. Schulz, P. F. Dittner, J. P. Giese, Q. C. Kessel, H. F. Krause, P. D. Miller, and C. R. Vane, Phys. Rev. A 51, 324 (1995).

[7] L. H. Andersen, P. Hvelplund, H. Knudsen, S. P. Moller, J. O.P. Pedersen, E. Uggerhoj, K. Elsener, and E. Morenzoni, Phys. Rev. Lett. 62, 1731 (1989).

[8] S. P. Moller, E. Uggerhoj, H. Bluhme, H. Knudsen, U. Mikkelsen, K. Paludan, and E. Morenzoni, Phys. Rev. A 56, 2930 (1997).

[9] H. H. Andersen and J. Bottiger, Phys. Rev. A 7, 154 (1973).

[10] S. Datz, J. Gomes de Campo, P. F. Dittner, P. D. Miller, and J. A. Biggerstaff, Phys. Rev. Lett. 38, 1145 (1977).

[11] J. A. Golovchenko, A. N. Goland, J. S. Rosner, C. E. Thorn, H. E. Wegner, H. Knudsen, and C. D. Moak, Phys. Rev. B 23, 957 (1981); J. A. Golovchenko, D. E. Cox, and A. N. Goland, ibid. 26, 2335 (1982).

[12] G. de M. Azevedo, P. L. Grande, M. Behar, J. F. Dias, and G. Schiwietz, Phys. Rev. Lett. 86, 1482 (2001).
[13] L. L. Araujo, P. L. Grande, M. Behar, J. F. Dias, J. H.R. dos Santos, and G. Schiwietz, Nucl. Instrum. Methods Phys. Res. B 193, 172 (2002).

[14] J. H.R. dos Santos, M. Behar, P. L. Grande, and H. Boudinov, Phys. Rev. B 55, 13651 (1997).

[15] W. Jiang, R. Grötzschel, W. Pilz, B. Schmidt, and W. Möller, Phys. Rev. B 59, 226 (1999); 60, 714 (1999).

[16] A. L. Lifschitz and N. R. Arista (unpublished). The HISTOP (stopping power of heavy ions) program is freely available from the web page http://cabcat1.cnea.gov.ar/ colato/grupos/ stop/index.html.

[17] G. Schiwietz and P. L. Grande, Nucl. Instrum. Methods Phys. Res. B 153, 1 (1999); G. de M. Azevedo, P. L. Grande, and G. Schiwietz, ibid. 164, 203 (2000).

[18] P. L. Grande and G. Schiwietz, Nucl. Instrum. Methods Phys. Res. B 195, 55 (2002).

[19] J. H.R. dos Santos, P. L. Grande, M. Behar, H. Boudinov, and G. Schiwietz, Phys. Rev. B 55, 4332 (1997).

[20] A. Dygo, W. N. Lennard, and I. V. Mitchell, Nucl. Instrum. Methods Phys. Res. B 84, 23 (1994).

[21] L. L. Araujo, M. Behar, P. L. Grande, and J. F. Dias, Nucl. Instrum. Methods Phys. Res. B 219, 246 (2004).

[22] The same experimental procedure as in Ref. [21] but for B ions. The results are similar (to within 10\%) to those reported in Refs. [14,15].

[23] M. Deutsh, Phys. Rev. B 45, 646 (1992).

[24] P. Sigmund and A. Schinner, Nucl. Instrum. Methods Phys. Res. B 195, 64 (2002).

[25] G. de M. Azevedo, M. Behar, J. F. Dias, P. L. Grande, D. L. da 
Silva, and G. Schiwietz, Phys. Rev. B 65, 075203 (2002).

[26] W. Fritsch and C. D. Lin, Phys. Rep. 202, 1 (1991); J. F. Reading, T. Bronk, A. L. Ford, L. A. Wehrman, and K. A. Hall, J. Phys. B 30, L189 (1997).

[27] G. Schiwietz, Phys. Rev. A 42, 296 (1990); P. L. Grande and G. Schiwietz, ibid. 44, 2984 (1991); G. Schiwietz and P. L. Grande, Nucl. Instrum. Methods Phys. Res. B 69, 10 (1992); P. L. Grande and G. Schiwietz, Phys. Rev. A 47, 1119 (1993).

[28] A. F. Lifschitz and N. R. Arista, Phys. Rev. A 57, 200 (1998); 58, 2168 (1999); 59, 2719 (1999).

[29] N. R. Arista, Nucl. Instrum. Methods Phys. Res. B 195, 91 (2002).

[30] Program CASP (convolution approximation for swift particles) available at http://www.hmi.de/people/schiwietz/casp.html

[31] F. Bloch, Ann. Phys. (Leipzig) 16, 285 (1933).

[32] Shell corrections have been implemented according to Ref.
[24] in the CASP [30] 2.3 version.

[33] J. H. Barret, Phys. Rev. B 3, 1527 (1971).

[34] J. Bang and J. M. Hansteen, K. Dan. Vidensk. Selsk. Mat. Fys. Medd. 31, No. 13 (1959); L. Wilets and S. J. Wallace, Phys. Rev. 169, 84 (1968); M. R. Flannery and K. J. MacCann, Phys. Rev. A 8, 2915 (1973).

[35] P. L. Grande and G. Schiwietz, Nucl. Instrum. Methods Phys. Res. B 136-138, 125 (1998).

[36] F. H. Eisen, G. J. Clark, J. Bottiger, and J. M. Poate, Radiat. Eff. 13, 93 (1972).

[37] G. Lulli, E. Albertazzi, M. Bianconi, G. G. Bentini, R. Nipoti, and R. Lotti, Nucl. Instrum. Methods Phys. Res. B 170, 1 (2000).

[38] J. Lindhard, Nucl. Instrum. Methods 132, 1 (1976).

[39] J. M. Pitarke and I. Campillo, Nucl. Instrum. Methods Phys. Res. B 164-165, 147 (2000). 